\title{
Dysfunction of the histone demethylase IBM1 in Arabidopsis reshapes the root-associated bacteria community
}

\author{
Suhui Lv ${ }^{1.2}$, Yu Yang ${ }^{1}$, Li Peng ${ }^{1}$, Kai Tang ${ }^{3}$, Richa Kaushal ${ }^{1}$, Huiming Zhang $^{1 *}$ \\ ${ }^{1}$ Shanghai Center for Plant Stress Biology, CAS Center for Excellence in Molecular Plant Sciences, \\ Chinese Academy of Sciences, Shanghai 201602, China \\ ${ }^{2}$ University of Chinese Academy of Sciences, Beijing 100049, China \\ ${ }^{3}$ Department of Horticulture \& Landscape Architecture, Purdue University, West Lafayette, IN, \\ USA. \\ *Correspondence: H. Zhang (hmzhang@sibs.ac.cn)
}

\begin{abstract}
Root-associated bacteria communities are influential to plant growth and stress tolerance. Epigenetic regulation plays important roles in many plant biological processes, but its potential impacts on the assembly of root microbiota remain unclear. Here we report that dysfunction of the histone demethylase IBM1 in Arabidopsis substantially alters root-associated soil bacteria community. We compared two alleles of ibm1 mutant (ibm1-1 and ibm1-4) with wild type Arabidopsis regarding the root-associated bacteria community by 165 rRNA gene sequencing. The constrained principal coordinate analysis ( $P C O A)$ showed that the $i b m 1$ mutants are both clearly separated from Col-0 along the major coordinates. Among the 29 families which have a relative abundance more than $0.5 \%$ in at least one sample, 10 and 11 families were commonly affected by ibm1-1 and ibm1-4 alleles in the rhizosphere and the endosphere compartment, respectively. Notably, the ACMs (Abundant Community members) belonging Pseudomonadaceae showed increased relative abundance in the $i b m 1$ mutant alleles compared to Col- 0 in both the rhizosphere and the endosphere compartments. The ACMs belonging to Oxalobacteraceae mostly showed decreased relative abundance in $i b m 1$ mutants compared to Col- 0 in endosphere compartment. These findings demonstrate an influential function of IBM1-mediated epigenetic regulation in shaping the root-associated microbiota.
\end{abstract}

\section{Introduction}

The plant rhizosphere, which refers to a thin layer of soil adhering to the roots, harbors various microorganisms especially bacteria in terms of species richness (1). While some rhizobacteria have no observable effects on plants, others are either pathogens that cause detrimental effects on plants or beneficial strains termed plant growth-promoting rhizobacteria (PGPR) that promote plant vigor $(1,2)$. The root-associated bacteria community has been shown to be important for plants health (3), and is therefore emerging as an important target for soil management and for studying plant-microbe interactions. 
The plant immune response plays an important role in sculpting the plant-associated microbiome. Evidences have shown that plant-associated microbiome can be changed when plants are attacked by pathogen or insect (3-5). For example, foliar pathogen Pseudomonas syringae pv tomato (Pst DC3000) induces the Arabidopsis root secretions of L-malic acid (MA), which selectively recruits the beneficial rhizobacterium Bacillus subtilis FB17 (6). For another example, aphid (Myzus persicae) infestation on pepper plants increased the root population of the beneficial Bacillus subtilis GBO3, but reduced population of the pathogenic Ralstonia solanacearum SL1931 (7). Thus, it seems that plants are able to recruit beneficial microorganisms in the rhizosphere to help suppress pathogens through immune response upon attacked. Besides, analysis of Arabidopsis root microbiota using mutants which either constitutively accumulates defense phytohormone salicylic acid (SA) or impaired in SA accumulation/signaling have revealed that SA modulates colonization of the root by specific bacterial families (8). Moreover, the Arabidopsis quadruple mutant ( $\min 7$ fs2 efr cerk1; hereafter, $m f e c)$, simultaneously defective in pattern-triggered immunity and the MIN7 vesicle-trafficking pathway, also harbors altered endophytic phyllosphere microbiota (9). These findings demonstrate that plant immunity system functions in shaping plant associated microbiome.

In the past decade, the role of epigenetic factors in controlling plant immunity has been emerging. Several studies have implicated that DNA demethylation functions in plant immune response (10-14). For example, the triple DNA demethylase mutant, rdd (ros1 dm/2 dm/3), shows increased susceptibility to the fungal pathogen Fusarium oxysporum, accompanied with decreased expression of many stress response genes(11). Further investigations indicate that DNA demethylase target promoter transposable elements to positively regulate stress-responsive genes (11). Meanwhile, RdDM component nrpe1 displayed enhanced resistance to the biotrophic pathogen Hyaloperonospora arabidopsidis ( $H p a$ ), which is associated with elevated SA-dependent PR1 gene expression upon Hpa infection(12). Moreover, global disruption of DNA methylation by met1 or ddc mutation activates defense responses against $P$. syringae (13). Interestingly, plants exposed to bacterial pathogen, avirulent bacteria, or SA hormone showed numerous stress-induced differentially methylated regions, many of which were intimately associated with differentially expressed genes (13). Some TEs are demethylated and transcriptionally reactivated during antibacterial defense in Arabidopsis, and DNA demethylation restricts multiplication and vascular propagation of the bacterial pathogen Pseudomonas syringae in leaves (14). These evidences indicate that DNA demethylation acts as part of the plant immune response. Besides DNA demethylation, histone modifications and chromatin remodeling also take part in plant immunity control. For example, an Arabidopsis JHDM2 (JmjC domain-containing histone demethylase 2) family protein JMJ27, which displays H3K9me1/2 demethylase activity, is induced in response to virulent Pseudomonas syringae pathogens and is required for resistance against these pathogens (15). For another example, H2A.Z is the variant of the canonical histone H2A, and SWR1-like complex is responsible for the H2A.Z deposition (16). 
Mutation of H2A.Z coding genes (hta9 hta11) or SWR1 complex components (pie1, sef) caused constitutive expression of systemic acquired resistance (SAR) marker genes and increased resistance to the virulent Pseudomonous syringae (16). Altogether, it is evident that epigenetic regulation, including DNA (de)methylation, histone modification, and chromatin remodeling, plays an important role in plant immune response.

Arabidopsis IBM1 (increase in bonsai methylation 1) encodes a Jmjc-domain containing $\mathrm{H} 3 \mathrm{~K} 9$ demethylase, mutation of which resulted in ectopic H3K9me and non-CG methylation in thousands of genes, as well as a variety of developmental phenotypes (17-20). The ibm1-induced genic H3K9me2 depends on both histone methylase KYP/SUVH4 and DNA methylase CMT3 (20). Later it was found that developmental defects of $i b m 1$ can be suppressed by a second mutation in $L D L 2$ (LYSINE-SPECIFIC DEMETHYLASE 1-LIKE 2) gene, a homolog of H3K4 demethylase (21). Interestingly, the IdI2 mutation suppressed the developmental defects without suppressing the $i b m 1$-induced ectopic H3K9me2 (21). It was proposed that the ectopic H3K9me2 mark directed removal of gene-body H3K4me1 and caused transcriptional repression in an LDL2-dependent manner (21). In another study, it was shown that the ibm1 mutation caused hypermethylation of H3K9 and DNA non-CG sites within RDR2 and DCL3, which are two components in the RNA-directed DNA methylation (RdDM) pathway. The down-regulation of RDR2 and DCL3 gene expression in ibm1 affected siRNA biogenesis in a locus-specific manner and disrupted RdDM-directed gene repression. (22). Thus dysfunction of IBM1 has a strong impact on plant epigenetic landscape including histone modifications and DNA methylation patterns.

In this study, we compared two alleles of $i b m 1$ mutant with wild type Arabidopsis regarding the root-associated bacteria community. The 16S rRNA gene sequencing results revealed substantial impacts of the $i b m 1$ mutations on the bacteria communities in the rhizosphere and endosphere. Particularly, we show that the Pseudomonadaceae family is enriched in the $i b m 1$ mutant alleles compared to Col-0 in both rhizosphere and endosphere compartments, while the Oxalobacteraceae family showed decreased relative abundance in $i b m 1$ mutants compared to Col- 0 in endosphere compartment. Our study of IBM1 demonstrates an influential function of epigenetic regulation in shaping the root-associated microbiota.

\section{Results}

\section{Ibm1 mutation substantially alters plant root-associated microbiota}

To evaluate the potential effects of $i b m 1$ mutation on the plant-bacteria interaction, we investigated the rhizosphere and root endosphere microbiomes of $\mathrm{Col}-\mathrm{O}$ and two $i b m 1$ mutant lines (ibm1-1 and ibm1-4). We collected field soil from Chenshan Botanical Garden, Shanghai, China, and grew the Col-0, ibm1-1 and ibm1-4 in this field soil. 17 days later, the DNA were extracted from bulk soil, rhizosphere and 
endosphere compartments of different genotypes. Then the bacterial 16S rRNA gene amplicon libraries were generated with the PCR primers 799F and 1193R harboring the hypervariable regions V5-V6-V7 $(23,24)$, and the bacteria communities were profiled by $16 \mathrm{~s}$ rDNA sequencing.

A total of $1,847,563$ high-quality sequences were obtained with a median read count per sample of 57,784 (range 7,511-137,080) across all the 35 samples. To correct the sequencing depth differences among samples, these high-quality sequences were further subjected to rarefaction to 7000 sequences per sample based on the rarefaction curve (Figure 1A), which resulted in 4688 unique OTUs as the threshold-independent community (TIC) (Dataset S1). A minimum of 20 sequences per OTU in at least one sample was used as a criterion to define abundant community members (ACMs). The ACM of all samples was represented by 186 bacterial OTUs comprising of $66.73 \%$ of the rarefaction quality sequences (Dataset S1). We normalized this table by dividing the reads per OTU in a sample by the sum of the usable reads in that sample, resulting in a table of relative abundances (RA) (Dataset S1) for quantitative comparison.

We first examined taxonomic composition in the whole dataset. The bulk soil samples are mainly composed of Proteobacteria and Bacteroidetes, with smaller portions of Acidobacteria (4.6\%), Verrucomicrobia (4.4\%), Nitrospirae (4.0\%), Gemmatimonadetes $(0.61 \%)$, Firmicutes $(0.40 \%)$, Actinobacteria $(0.22 \%)$, and

Chloroflexi (0.11\%) (Figure 1B ; Dataset S1). Meanwhile, the rhizosphere and root communities were both dominated by Proteobacteria and Bacteroidetes regardless of genotypes (Figure 1B). Then we used the weighted UniFrac metric to compare community diversity between samples (25) (Figure $1 \mathrm{C}$ ). Consistent with previous studies (26-28), the hierarchical clustering of UniFrac distances revealed that the compartments (bulk soil, rhizosphere, endosphere) are the major sources of variation in bacteria communities.

Next, we performed constrained principal coordinate analysis (PCOA) of ACM to evaluate the impacts of $i b m 1$ mutation on bacteria communities in the rhizosphere and the endosphere. The $i b m 1$ mutants are both clearly separated from Col-0 along the major coordinates, which represent $79 \%$ and $73 \%$ variances for the rhizosphere and the endosphere, respectively (Figure $2 \mathrm{~A}$ and $\mathrm{B}$ ), indicating that IBM1 dysfunction alters the microbial communities in these two compartments.

To some extent, the ibm1-1 and ibm1-4 alleles are also separated to each other along the major coordinates (Figure $2 \mathrm{~A}$ and $\mathrm{B}$ ), which may result from their different mutation types. Thus, we only consider those bacteria communities which were commonly affected in ibm1-1 and ibm1-4 as the "ibm1-affected" bacteria communities $(\mathrm{P}<0.05)$. At the phylum level, Firmicutes were enriched in both ibm1-1 and $i b m 1-4$ rhizosphere compartments, and Verrucomicrobia were depleted in both 
ibm1-1 and ibm1-4 endosphere compartments (Dataset S1). However, only 1 ACM belonging to Firmicutes was identified as significantly enriched in ibm1 mutants; and only $1 \mathrm{ACM}$ belonging to Verrucomicrobia was identified as significantly depleted in $i b m 1$ mutants (Datset S1). Then we looked into the "ibm1-affected" bacteria communities at family level (Dataset S1, Figure 2C and D). In rhizosphere compartment, 10 of 29 families (RA > 5\%) were commonly affected in both ibm1 mutant alleles $(\mathrm{P}<0.05)$, among which 4 are enriched and 6 are decreased in ibm1 mutants (Figure 1C). The 4 families enriched in ibm1 mutants include Pseudomonadaceae, Enterobacteriaceae, Bacillaceae, and Aeromonadaceae; while the 6 families with decreased relative abundance in $i b m 1$ mutants are Caulobacteraceae, Rhizobiaceae, Sinobacteraceae, Sphingomonadaceae, Oxalobacteraceae and a "not_assigned" family (Figure 2C; Dataset S1). In endosphere compartment, 11 families are commonly affected in both ibm1-1 and ibm1-4 alleles, among which 4 are enriched (Comamonadaceae, Pseudomonadaceae, Enterobacteriaceae, and Aeromonadaceae) in the ibm 1 mutants, and 7 showed decreased relative abundance (Sinobacteraceae, Oxalobacteraceae, Caulobacteraceae, Sphingomonadaceae, Xanthomonadaceae, Opitutaceae and a "not assigned" family) in ibm1 mutants (Figure 2D; Dataset S1).

At the ACM level, among 164 ACMs with a relative abundance more than $5 \%$, 35 were commonly affected by $i b m 1-1$ and $i b m 1-4$ in rhizosphere compartment, while 36 were commonly affected by $i b m 1-1$ and $i b m 1-4$ in endosphere compartment (Dataset S1). In rhizosphere, 14 of the ibm1-affected ACMs are upregulated in the $i b m 1$ mutants, of which 6 belong to Pseudomonadaceae, 1 belongs to Aeromonadaceae, 3 belong to Comamonadaceae, 1 belongs to Flavobacteriaceae, 2 belong to Enterobacteriaceae and 1 belong to Bacillaceae (Figure S2A). Meanwhile, 21 of the ibm1-affected rhizosphere ACMs are downregulated in $i b m 1$ mutants, including ACMs belonging to Rizobeaceae (3), Flavobacteriaceae (3), Comamonadaceae (3), Xanthomonadaceae (1), Oxalobacteraceae (2), Sphingomonadaceae (1) Sinobacteraceae (1), Caulobacteraceae (2) and 5 ACMs with "not assigned" family (Figure S2B). In endosphere, 13 of the ibm1-affected ACMs are upregulated in the $i b m 1$ mutants, including 4 belonging to Pseudomonadaceae, 1 belonging to Aeromonadaceae, 5 belonging to Comamonadaceae, 1 belonging to Rizobeaceae, 1 belonging to Methylophilaceae, 1 belonging to Enterobacteriaceae (Figure S2C). 23 of the ibm1-affected ACMs are downregulated in the ibm 1 mutants, including 6 from Oxalobacteraceae, 6 from Comamonadaceae, 2 from Caulobacteraceae, 1 from Cytophagaceae, 1 from Opitutaceae, 1 from Rhizobiaceae, 1 from Sinobacteraceae, 1 from Sphingomonadaceae, and 4 with "not assigned" family (Figure S2D). Altogether, these results revealed an influential role of IBM1 in the assembly of plant-associated bacteria community.

Notably, in both rhizosphere and endosphere compartments, the ACMs (RA>5\%o) 
belonging to the families of Pseudomonadaceae all showed increased relative abundance in the ibm1 mutant alleles compared to Col-0 (Figure 3A and B; Dataset S1). Besides, in endosphere compartment, ACMs belonging to Oxalobacteraceae mostly showed decreased relative abundance in ibm1 mutants compared to Col-0

(Figure 3C; Dataset S1). It was documented that Pseudomonadaceae family are enriched in the root microbiota of cpr5 and cpr1 mutants, which constitutively produce and accumulate SA contents; while Oxalobacteraceae family are enriched in the pad4 mutant, which is impaired in SA signaling (8). Thus there is a possible correlation between high SA level and plant association with Pseudomonadaceae and Oxalobacteraceae.

\section{Discussion}

A distinct pattern in ibm 1 microbiota is that the Pseudomonadaceae family are enriched in ibm1 rhizosphere and endosphere compartments, while Oxalobacteraceae family are depleted in the endosphere compartment. It was reported that SA modulates colonization of the root by specific bacterial families (8). From the published microbiota data, we found that Pseudomonadaceae family are enriched in the root microbiota of cpr5 and cpr1 mutants, which constitutively produce and accumulate SA contents; and Oxalobacteraceae family are enriched in the pad4 mutant, which is impaired in SA signaling (8). These indicate a role of increased $S A$ in affecting the Pseudomonadaceae and Oxalobacteraceae root colonization in $i b m 1$ mutant. In addition to SA, other factors probably also contribute to shaping the ibm1 root microbiota. For example, flavonoids and coumarins are known Arabidopsis root exudates (29) which may affect the root-associated microbiota. In future, the root exudates profiles of $i b m 1$ may be necessary to explain how the root-associated microbiota changed in $i b m 1$ mutant.

\section{Methods}

\section{Plant material and growth condition}

Soil from the field in Shanghai Chen Shan Botanical Garden was collected, homogenized and mixed with commercial soil (Pindstrup Substrate) with 2:1 ratio, and placed into $10 \times 10 \times 8 \mathrm{~cm}$ pots. Then 14-day-old of Col-0, ibm1-1 and ibm1-4 seedlings were transplanted into the pots (five seedlings for each pot), and grown for another 17 days in a growth room at $22^{\circ} \mathrm{C}$ under $16 \mathrm{~h}$ light/8h dark condition. 5 biological replicates were prepared for each genotype and each biological replicate contains 10 plants. Unplanted pots were subjected to the same conditions as the planted pots to prepare the control soil samples at harvest.

\section{DNA sample preparation}

The rhizosphere samples and root endophyte samples were harvested according to Schlaeppi et al. (28). DNA were extracted from those samples by FastDNA ${ }^{\circledast}$ SPIN kit for Soil (MP Biomedicals). Samples were homogenized in the Lysis Matrix E tubes using a Retsch MM400 mill at a frequency of $30 \mathrm{~Hz}$ for 30 seconds. DNA samples 
were eluted in 50-100 $\mu$ DES water and DNA concentrations were determined using the Qubit ${ }^{\mathrm{TM}}$ dsDNA HS Assay Kit (Invitrogen, life technologies) on Qubit ${ }^{\circledR} 2.0$ (Life Technologies).

\section{Amplicon generation and library construction}

$16 \mathrm{~S}$ rRNA amplicon generation and library preparation were performed according to Liu et al.(33). Amplicon libraries were generated using the PCR primers 799F (AACMGGATTAGATACCCKG)(24), and 1193R (5'-ACGTCATCCCCACCTTCC-3' )(23). The first amplification was performed in a $25 \mu \mathrm{L}$ reaction volume, including $2.5 \mu \mathrm{l}$ microbial DNA ( $5 \mathrm{ng} / \mu \mathrm{l}), 5 \mu \mathrm{l}$ of $799 \mathrm{~F}-\mathrm{B}$ primer $(1 \mu \mathrm{M}), 5 \mu \mathrm{l}$ of $1193 \mathrm{R}-\mathrm{B}$ primer $(1 \mu \mathrm{M})$, 12.5 $\mu \mathrm{l} 2 \mathrm{X} \mathrm{KAPA} \mathrm{HiFi} \mathrm{Hot} \mathrm{Start} \mathrm{Ready} \mathrm{Mix.} \mathrm{The} \mathrm{PCR} \mathrm{setting} \mathrm{was} 95^{\circ} \mathrm{C}$ for 3 minutes, 30 cycles of $95^{\circ} \mathrm{C}$ for 30 seconds, $55^{\circ} \mathrm{C}$ for 30 seconds, $72^{\circ} \mathrm{C}$ for 30 seconds, and $72^{\circ} \mathrm{C}$ for 5 minutes. The second amplification was conducted in $20 \mu \mathrm{L}$ reaction volume, each containing $10 \mu \mathrm{L} 2 \mathrm{x}$ KAPA HiFi Hot Start Ready Mix, $200 \mathrm{nM}$ of 2P-F and 2P-R primer, $2 \mathrm{nM}$ of $\mathrm{F}-(\mathrm{N})$ and $\mathrm{R}-(\mathrm{N})$ primer, and 40 ng first-round $\mathrm{PCR}$ product. The $\mathrm{PCR}$ conditions were $95^{\circ} \mathrm{C}$ for 3 minutes, 10 cycles of $95^{\circ} \mathrm{C}$ for 30 seconds, $55^{\circ} \mathrm{C}$ for 30 seconds, $72^{\circ} \mathrm{C}$ for 30 seconds, and $72^{\circ} \mathrm{C}$ for 2 minutes. All the primers sequences were included in the Table S1.

After each PCR, the PCR products were loaded on $2 \%$ agarose gel and cut from the gel with Tanon UV-2000 gel imaging system and extracted from the agarose using the QIAquick Gel Extraction kit (Qiagen). DNA concentrations were determined using the Qubit $^{\text {TM }}$ dsDNA HS Assay Kit on Qubit ${ }^{\boxplus 2}$ 2.0.

After two-round PCR, 5 biological replicates for each genotype were combined, and DNA concentrations were determined using the Qubit ${ }^{\mathrm{TM}}$ dsDNA HS Assay Kit on Qubit ${ }^{\circledR}$ 2.0. Then all the genotype samples were combined with same amount of each genotype PCR product.

\section{Sequencing and data analysis}

The libraries were sent to Novogene company for 16s rRNA gene sequencing. Processing and statistical analysis of 16S rRNA counts was performed by the Core Facility of Bioinformatics in Shanghai Center for Plant Stress Biology, China.

The quality of reads was checked with fastqc $\vee 0.11 .7$ and the data was pre-processed with trimmomatics v0.39 (34). The processed high-quality data was assembled with FLASH v1.2.11(35). QIIME (version 1.9.1) was mainly used for subsequent analysis(36). Assembled reads were demultiplexed with split_library_fasta.py and chimeric sequences were removed by identify_chimera_seqs.py with usearch (-m usearch61) method and "Gold" database (-r gold.fa). Open-reference OTU picking was carried out using the pick_open_reference_otus.py script in QIIME at $97 \%$ sequence identity with GreenGenes 16S database v.13_8. After removing OTUs which belong to mitochondria, Chlorophyta, Archaea and Cyanobacteria, we got 6275 OTUs. The 
beta-diversity analysis and statistical analysis were performed mainly according to Schlaeppi et. al (28). We generated the rarefied tables (100x tables from 700-70000 sequences per sample, step of 700 sequences), selected $\# 50$ of rarefied OTU tables as the threshold-independent community (TIC) table. A minimum of 20 sequences per OTU in at least one sample was used as a criterion to define Abundant Community members $(A C M)(28)$. The relative abundance of ACM was calculated by dividing the reads per $A C M$ in a sample by the sum of the usable reads in that sample. Pairwise UniFrac distance and principal coordinates analysis were performed by QIIME. The significant differences between samples were assessed by the ANOVA-based statistics. The resulting $\mathrm{p}$-values were adjusted by Benjamini-Hochberg false discovery rate (FDR) correction for multiple hypotheses testing.

\section{Reference}

1. Mendes R, Garbeva P, \& Raaijmakers JM (2013) The rhizosphere microbiome: significance of plant beneficial, plant pathogenic, and human pathogenic microorganisms. FEMS Microbiol $\operatorname{Rev} 37(5): 634-663$.

2. Lugtenberg B \& Kamilova F (2009) Plant-growth-promoting rhizobacteria. Annu Rev Microbiol 63:541-556.

3. Berendsen RL, Pieterse CM, \& Bakker PA (2012) The rhizosphere microbiome and plant health. Trends Plant Sci 17(8):478-486.

4. Trivedi $\mathrm{P}$, et al. (2012) Huanglongbing alters the structure and functional diversity of microbial communities associated with citrus rhizosphere. ISME J 6(2):363-383.

5. Luan FG, et al. (2015) Analysis of microbial diversity and niche in rhizosphere soil of healthy and diseased cotton at the flowering stage in southern Xinjiang. Genet Mol Res 14(1):1602-1611.

6. Rudrappa T, Czymmek KJ, Pare PW, \& Bais HP (2008) Root-secreted malic acid recruits beneficial soil bacteria. Plant Physiol 148(3):1547-1556.

7. Lee B, Lee S, \& Ryu CM (2012) Foliar aphid feeding recruits rhizosphere bacteria and primes plant immunity against pathogenic and non-pathogenic bacteria in pepper. Ann Bot 110(2):281-290.

8. Lebeis SL, et al. (2015) PLANT MICROBIOME. Salicylic acid modulates colonization of the root microbiome by specific bacterial taxa. Science 349(6250):860-864.

9. Chen T, et al. (2020) A plant genetic network for preventing dysbiosis in the phyllosphere. Nature 580(7805):653-657.

10. Deleris A, Halter T, \& Navarro L (2016) DNA Methylation and Demethylation in Plant Immunity. Annu Rev Phytopathol 54:579-603.

11. Le TN, et al. (2014) DNA demethylases target promoter transposable elements to positively regulate stress responsive genes in Arabidopsis. Genome Biol 15(9):458.

12. Lopez Sanchez A, Stassen JH, Furci L, Smith LM, \& Ton J (2016) The role of DNA (de)methylation in immune responsiveness of Arabidopsis. Plant J 88(3):361-374.

13. Dowen $\mathrm{RH}$, et al. (2012) Widespread dynamic DNA methylation in response to biotic stress. Proc Nat I Acad Sci U S A 109(32):E2183-2191.

14. Yu A, et al. (2013) Dynamics and biological relevance of DNA demethylation in Arabidopsis antibacterial defense. Proc Natl Acad Sci U S A 110(6):2389-2394. 
15. Dutta A, Choudhary P, Caruana J, \& Raina R (2017) JMJ27, an Arabidopsis H3K9 histone demethylase, modulates defense against Pseudomonas syringae and flowering time. Plant $J$ 91(6):1015-1028.

16. March-Diaz R, et al. (2008) Histone H2A.Z and homologues of components of the SWR1 complex are required to control immunity in Arabidopsis. Plant $J$ 53(3):475-487.

17. Saze H, Shiraishi A, Miura A, \& Kakutani T (2008) Control of genic DNA methylation by a jmjC domain-containing protein in Arabidopsis thaliana. Science 319(5862):462-465.

18. Wang Y, Xue X, Zhu JK, \& Dong J (2016) Demethylation of ERECTA receptor genes by IBM1 histone demethylase affects stomatal development. Development 143(23):4452-4461.

19. Miura A, et al. (2009) An Arabidopsis jmjC domain protein protects transcribed genes from DNA methylation at CHG sites. Embo Journa/ 28(8):1078-1086.

20. Inagaki S, et al. (2010) Autocatalytic differentiation of epigenetic modifications within the Arabidopsis genome. Embo Journal 29(20):3496-3506.

21. Inagaki S, et al. (2017) Gene-body chromatin modification dynamics mediate epigenome differentiation in Arabidopsis. EMBO J 36(8):970-980.

22. Fan D, et al. (2012) IBM1, a JmjC domain-containing histone demethylase, is involved in the regulation of RNA-directed DNA methylation through the epigenetic control of RDR2 and DCL3 expression in Arabidopsis. Nucleic Acids Research 40(18):8905-8916.

23. Bodenhausen N, Horton MW, \& Bergelson J (2013) Bacterial communities associated with the leaves and the roots of Arabidopsis thaliana. PLOS ONE 8(2):e56329.

24. Chelius MK \& Triplett EW (2001) The Diversity of Archaea and Bacteria in Association with the Roots of Zea mays L. Microb Ecol 41(3):252-263.

25. Lozupone C \& Knight R (2005) UniFrac: a new phylogenetic method for comparing microbial communities. Applied and Environmental Microbiology 71(12):8228-8235.

26. Bulgarelli D, et al. (2012) Revealing structure and assembly cues for Arabidopsis root-inhabiting bacterial microbiota. Nature 488(7409):91-95.

27. Lundberg DS, et al. (2012) Defining the core Arabidopsis thaliana root microbiome. Nature 488(7409):86-90.

28. Schlaeppi K, Dombrowski N, Oter RG, Ver Loren van Themaat E, \& Schulze-Lefert P (2014) Quantitative divergence of the bacterial root microbiota in Arabidopsis thaliana relatives. Proc Natl Acad Sci U S A 111(2):585-592.

29. Monchgesang $S$, et al. (2016) Natural variation of root exudates in Arabidopsis thaliana-linking metabolomic and genomic data. Sci Rep 6:29033.

30. Voges M, Bai Y, Schulze-Lefert P, \& Sattely ES (2019) Plant-derived coumarins shape the composition of an Arabidopsis synthetic root microbiome. Proc Natl Acad Sci U S A 116(25):12558-12565.

31. Stringlis IA, et al. (2018) MYB72-dependent coumarin exudation shapes root microbiome assembly to promote plant health. Proc Natl Acad Sci U S A 115(22):E5213-E5222.

32. Rajniak J, et al. (2018) Biosynthes is of redox-active metabolites in response to iron deficiency in plants. Nat Chem Biol 14(5):442-450.

33. Liu Q et al. (2019) Hi-TOM: a platform for high-throughput tracking of mutations induced by CRISPR/Cas systems. Sci China Life Sci 62(1):1-7.

34. Bolger AM, Lohse M, \& Usadel B (2014) Trimmomatic: a flexible trimmer for Illumina sequence data. Bioinformatics 30(15):2114-2120. 
35. Magoc T \& Salzberg SL (2011) FLASH: fast length adjustment of short reads to improve genome assemblies. Bioinformatics 27(21):2957-2963.

36. Lawley B \& Tannock GW (Analysis of $16 \mathrm{~S}$ rRNA Gene Amplicon Sequences Using the QIIME Software Package. (1940-6029 (Electronic)).

Figure 1 [A] Rarefaction analysis. The pooling of the sequences from all the samples was permitted to display the bacterial communities with a sequencing depth of 7,511 - 137,080 quality sequences per sample. [B] Taxonomic structure at the phylum rank of the ACM. [C] Beta diversity. Between-sample diversity was calculated for ACM by weighted UniFrac distance metric on 7,000 sequences per sample.

Figure $2[A]$ and $[B]$ Constrained principal coordinate analysis (PCOA) of ACM in the rhizosphere $[\mathrm{A}]$ and endosphere $[\mathrm{B}]$ of Col-0, ibm1-1 and ibm1-4. The significant ACMs (fold change $>2$, FDR<0.05) were used for canonical analysis of principal coordinates, which was constrained for the genotypes. The percentage of variation explained by each axis refers to the fraction of the total variance of the data (ACM) explained by the constrained factor. [C] and [D] The families which are commonly affected by $i b m 1-1$ and $i b m 1-4(p<0.05)$ in rhizosphere $[C]$ and endoshphere [D], separately. FC: fold change.

Figure $3[A]$ and [B] Relative abundance (RA) of ACMs belonging to Pseudomonadaceae at rhizosphere $[A]$ and endosphere $[B]$, separately. $[C]$ Relative abundance of $A C M$ s belonging to Oxalobacteraceae at endosphere compartment. Values are mean $\pm S E, n=5$. Asterisks indicate significant differences between ibm 1 mutant and wild type Col-0 ( $p<0.05$, ANOVA).

Figure S1 Taxonomic structure at the family rank of the ACM in Col-0, ibm1-1 and ibm1-4 at rhizosphere $[A]$, endosphere $[B]$ and soil sample $[C]$.

Figure S2 The composition of the ACMs which are commonly affected by ibm1-1 and ibm1-4 $(p<0.05)$. The color in the pie chart represents the bacterial phyla of the corresponding taxa. The ACMs were displayed at order and family rank in the inner and the outer ring of the pie chart, respectively.

Table S1. Primers used in this study 
[A]

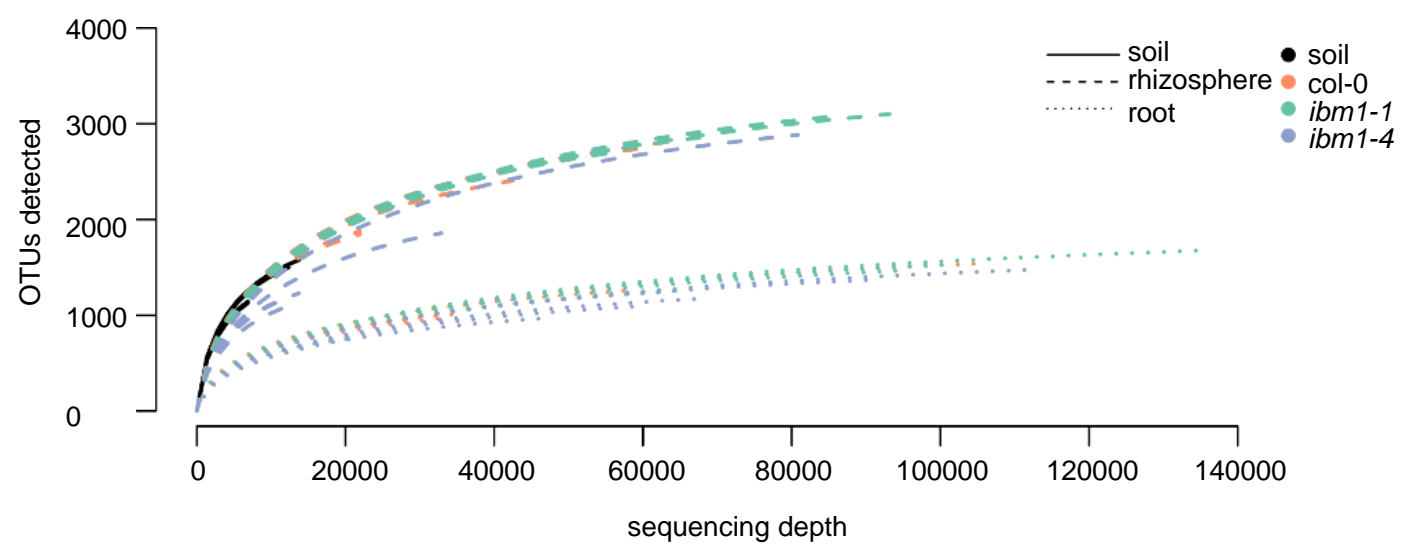

[B]
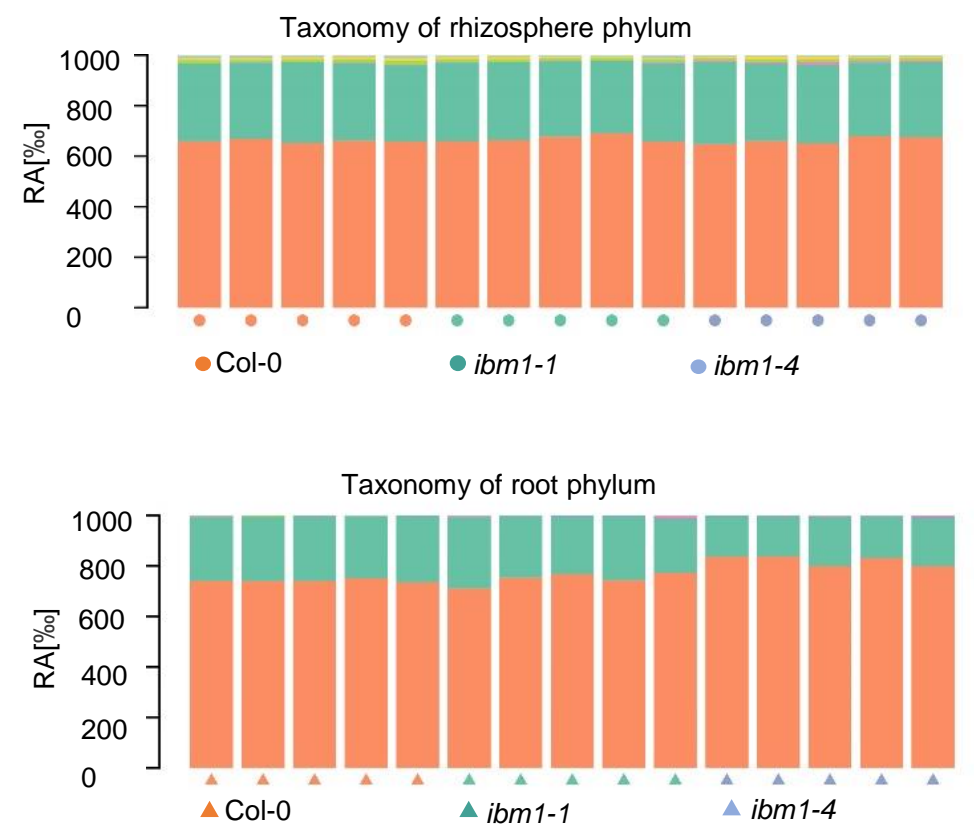

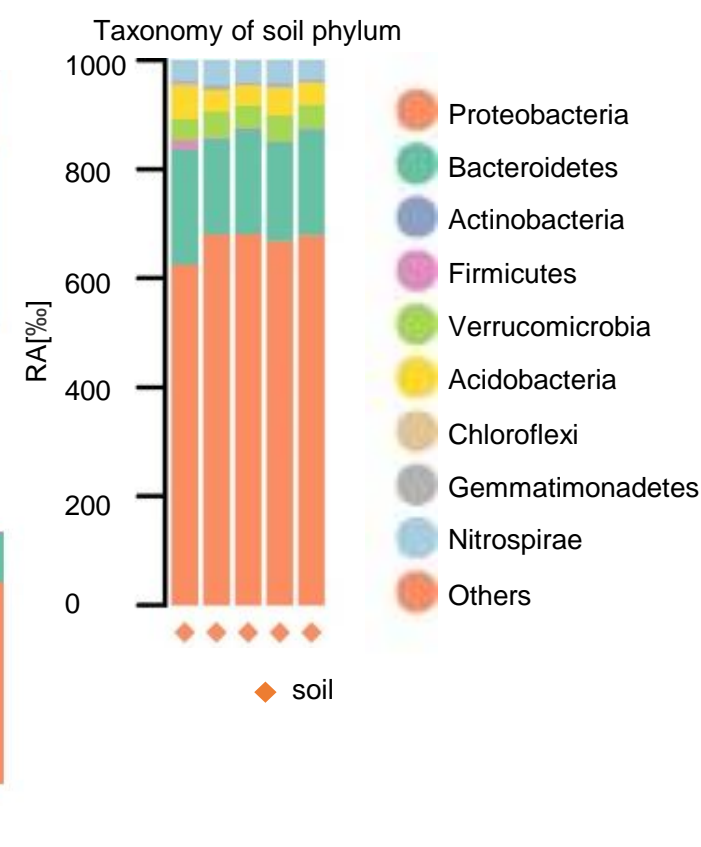

[C]
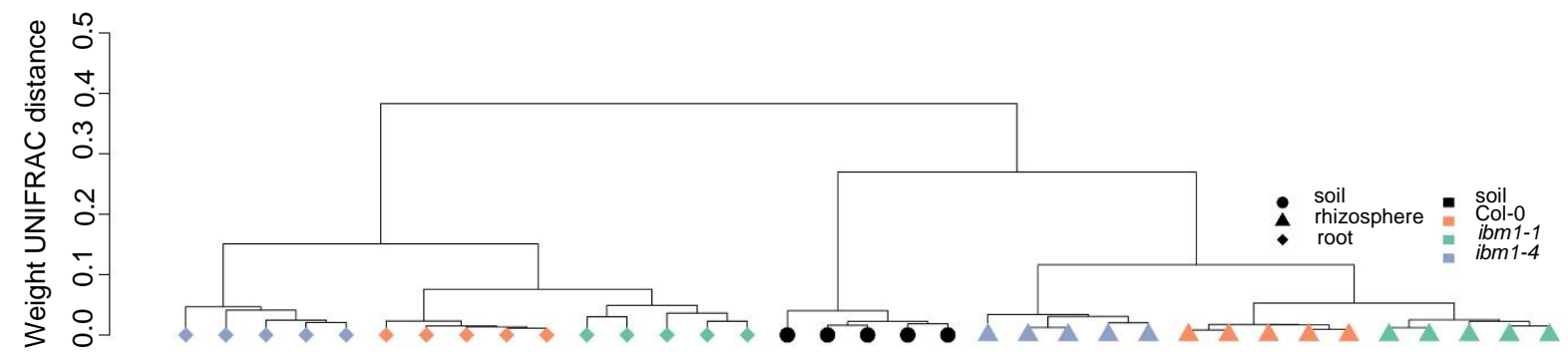

\section{Figure 1}


[B]

Rhizosphere

Species: [79\% of variance; $p<0.001 ; 95 \% \mathrm{Cl}=21 \%, 246 \%]$

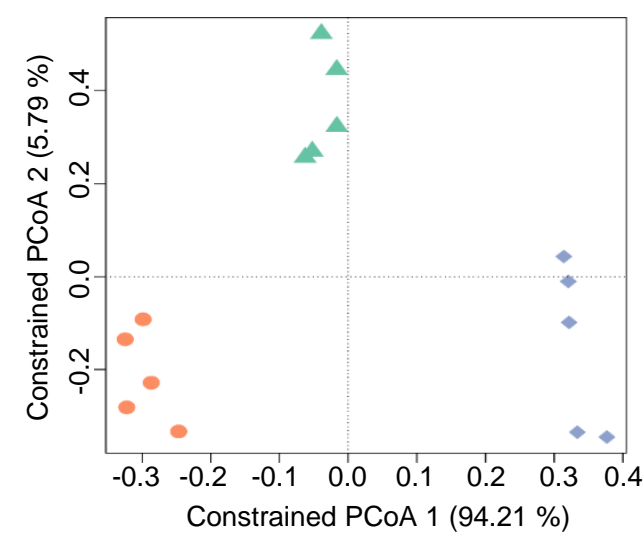

[C]

Rhizosphere

$\log _{10} \mathrm{FC}(\mathrm{ibm} 1 / \mathrm{Col}-0)$

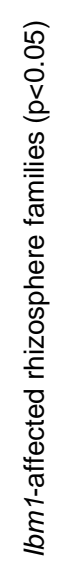

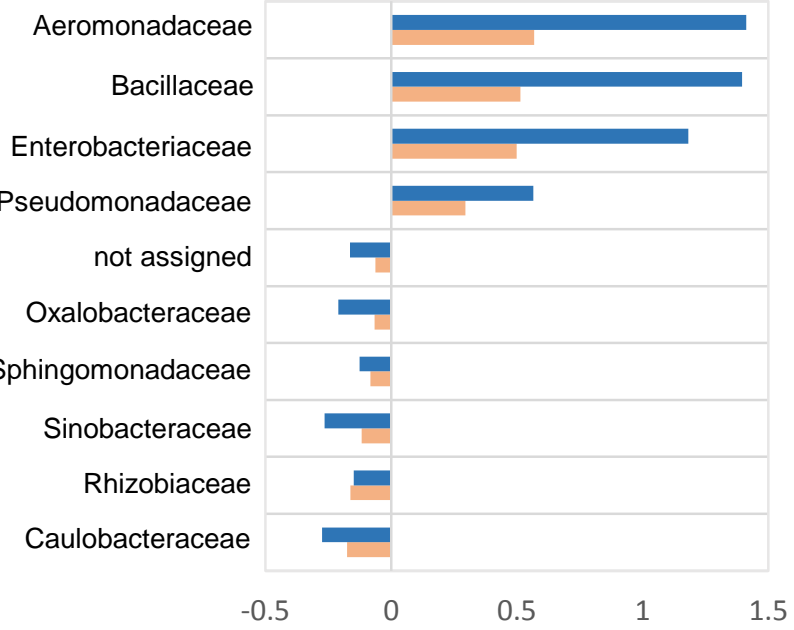

-ibm1-4 ibm1-1 ibm1-4 ibm1-1
Endosphere

Species: $[73 \%$ of variance; $p<0.001 ; 95 \% \mathrm{Cl}=23 \%, 218 \%]$

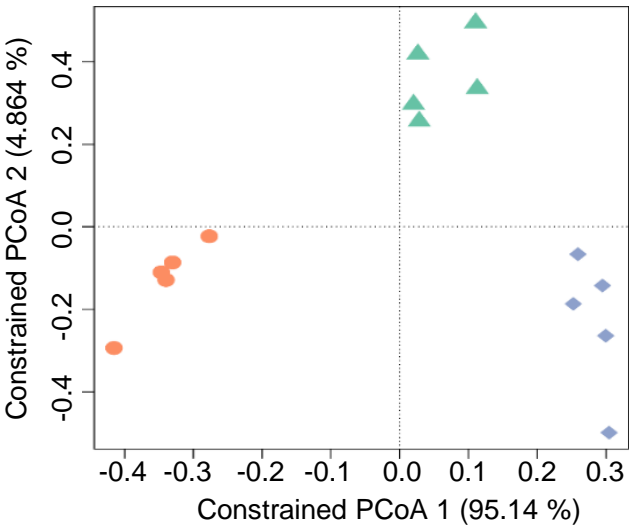

Col-0

ibm1-1

ibm1-4
[D]

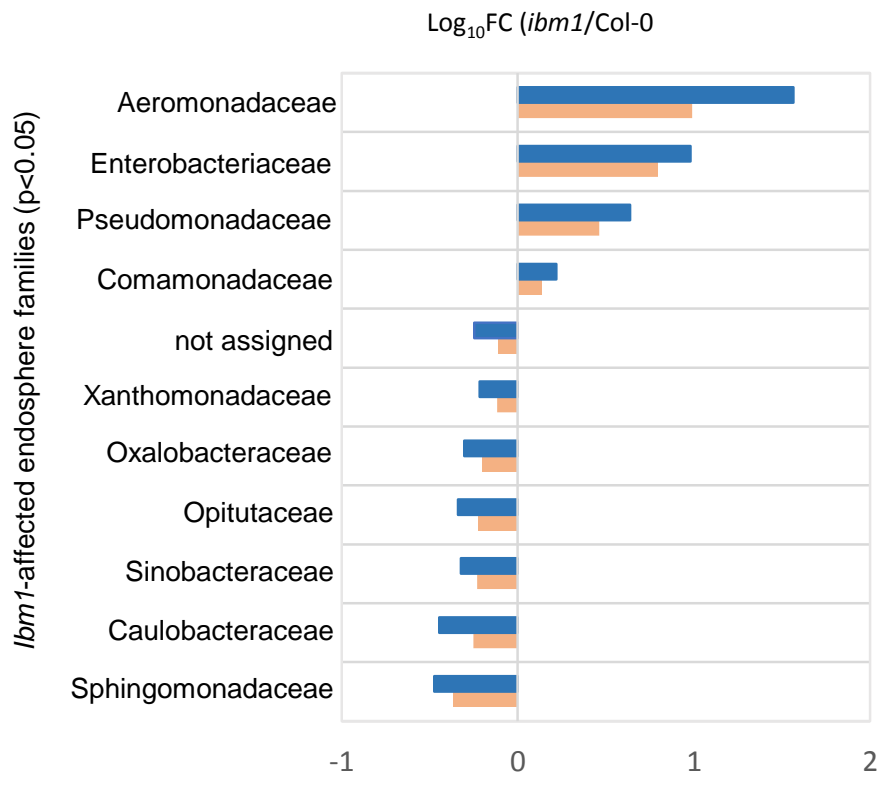

—ibm1-4 घibm1-1

\section{Figure 2}


[A]

Rhizosphere pseudomonas OTUs

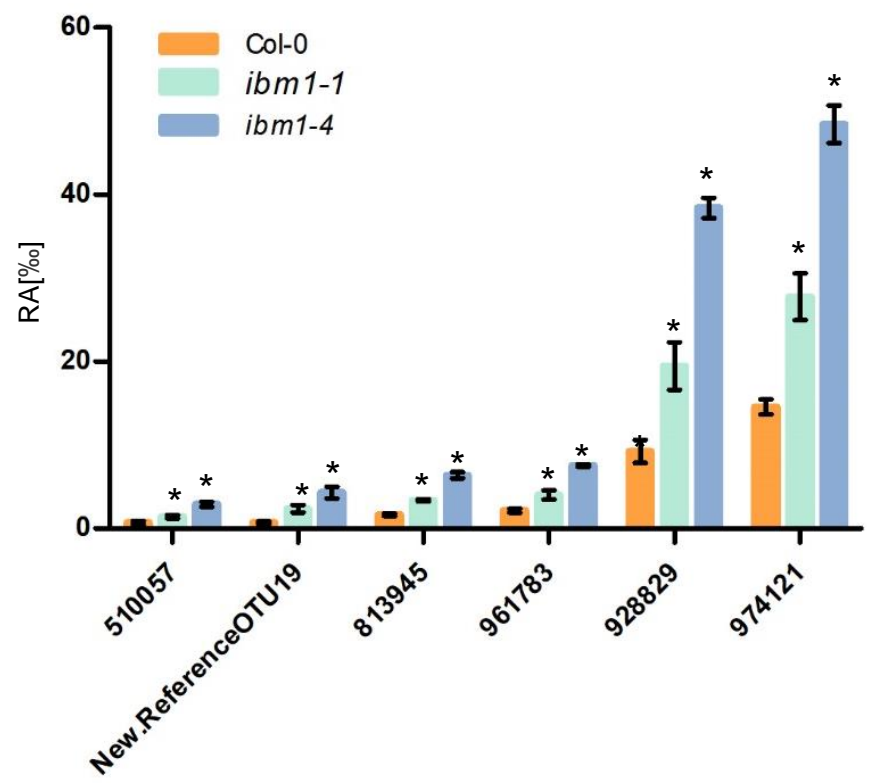

[C]

OTUs from root Oxalobacteraceae
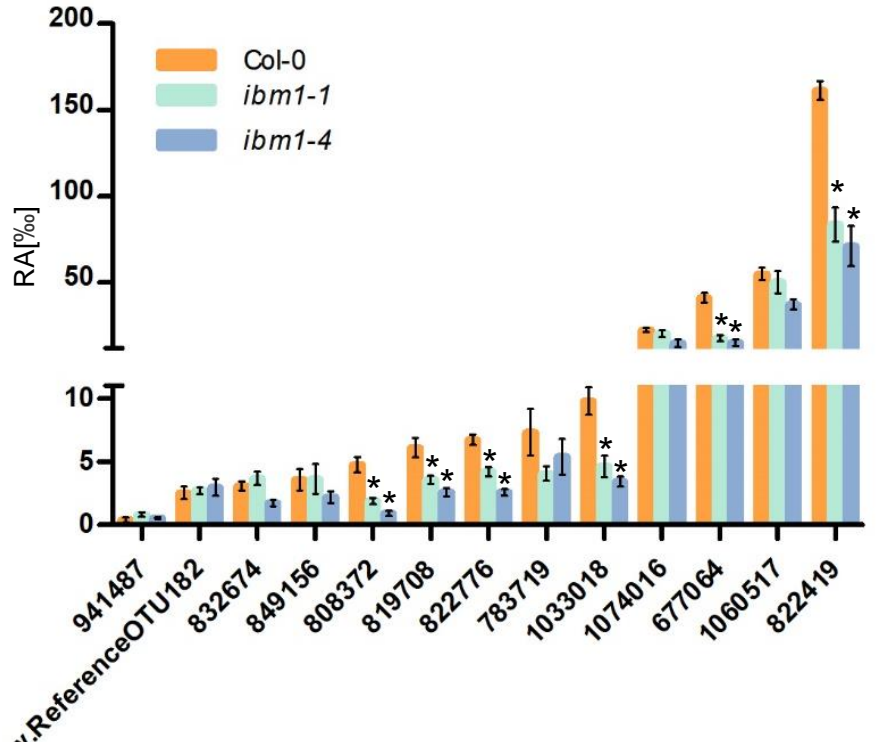

Figure 3
[B] Endosphere pseudomonas OTUs

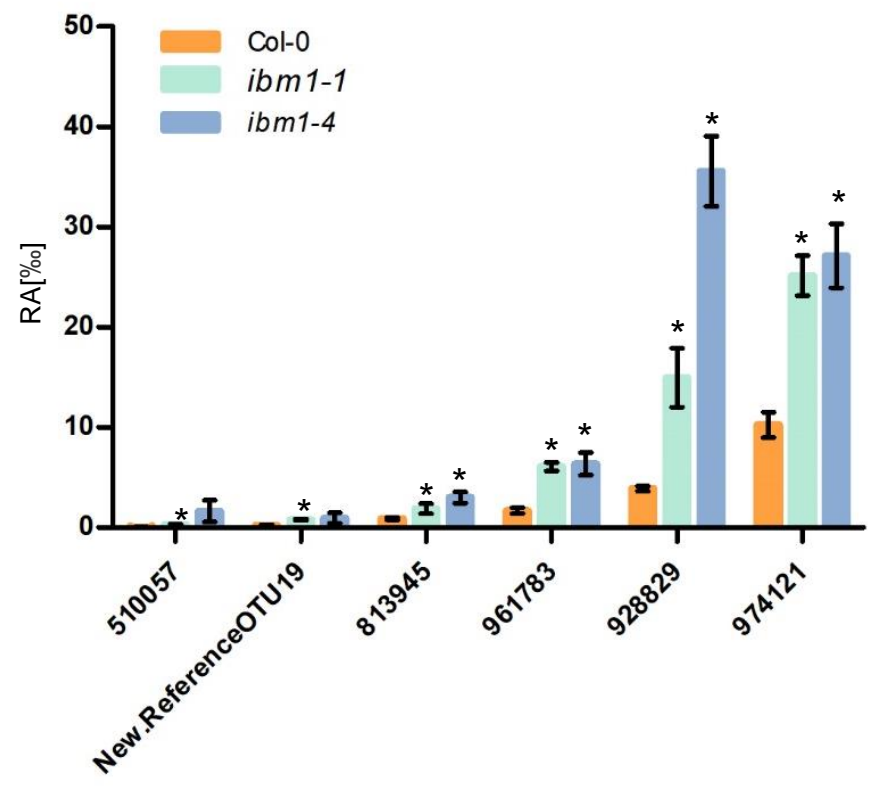


[A]

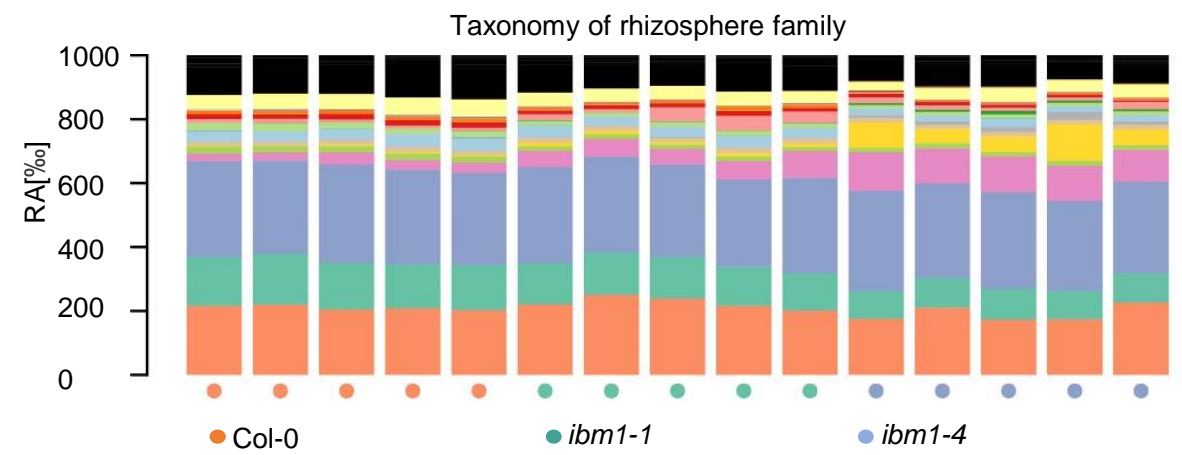

[B]

Taxonomy of root family

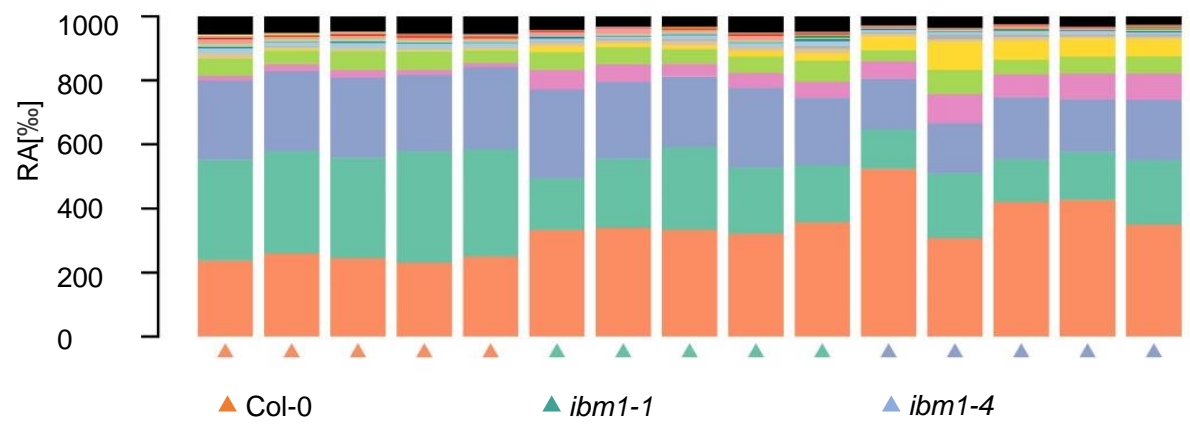

[C]

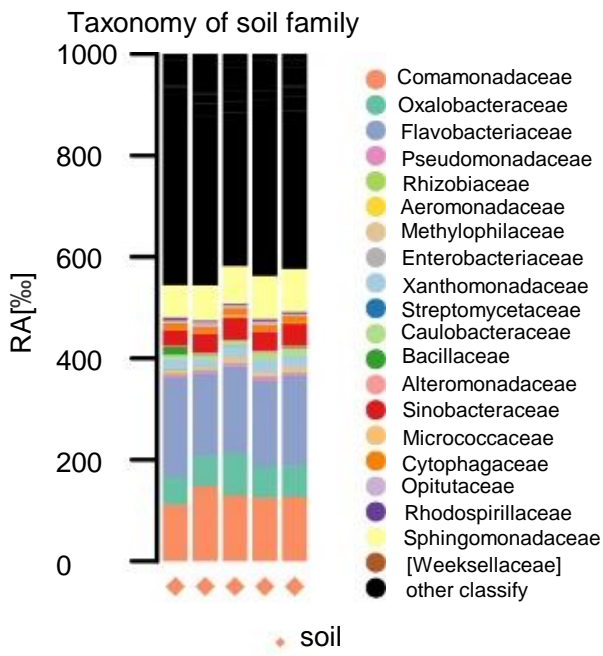

Figure S1 


\section{Rhizosphere up}

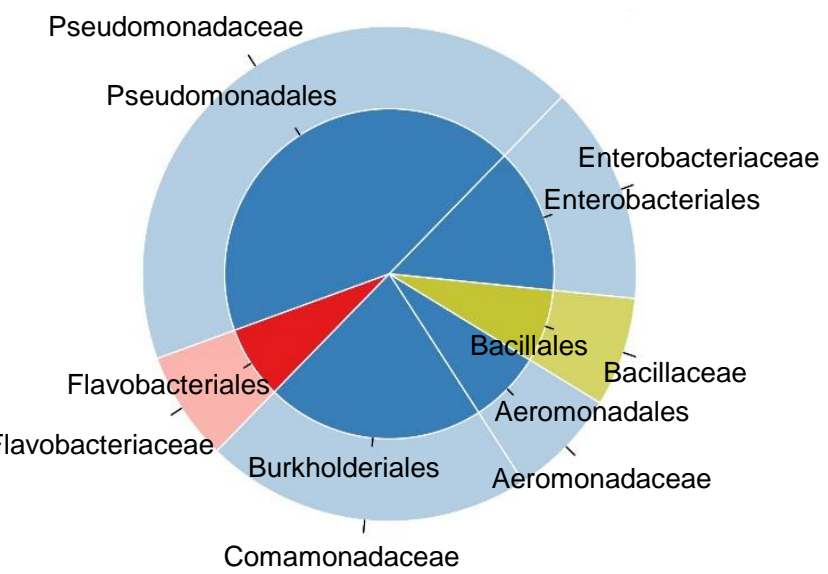

\section{Endosphere up}

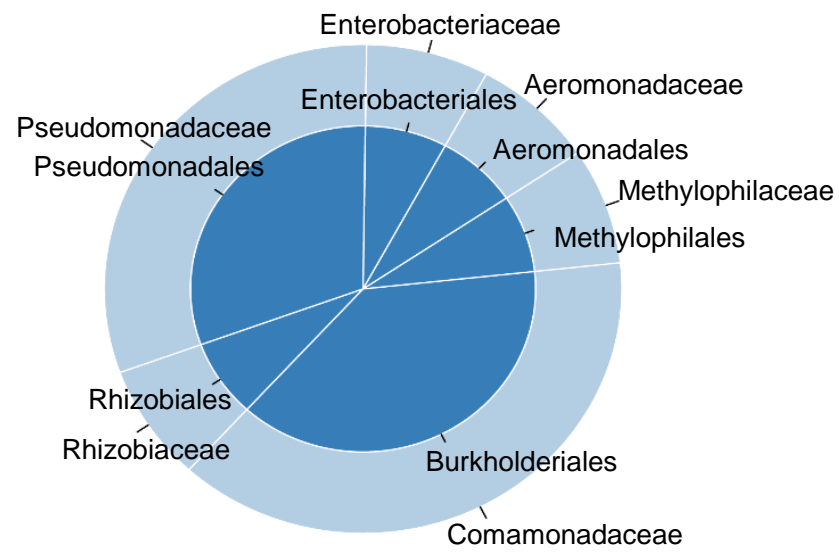

- Proteobacteria

- Bacteroidetes

Firmicutes

- Verrucomicrobia

\section{Rhizosphere down}

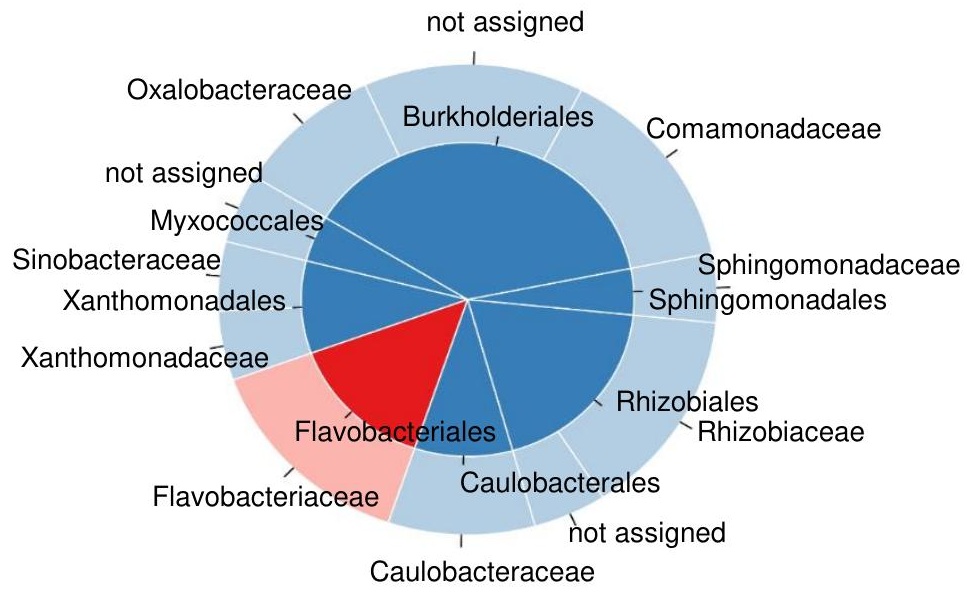

Endosphere down

Oxalobacteraceae

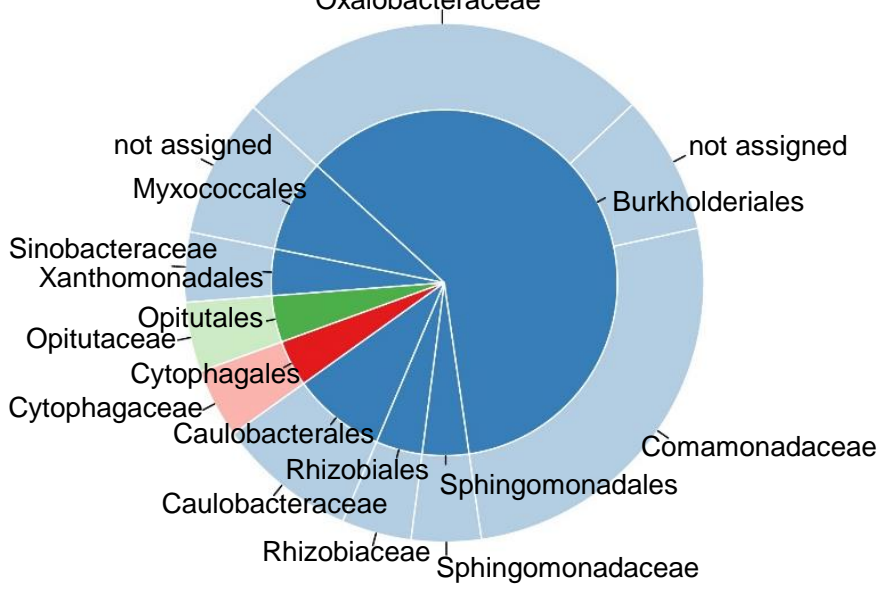

\section{Figure S2}

